\title{
Remifentanil Hydrochloride
}

National Cancer Institute

\section{Source}

National Cancer Institute. Remifentanil Hydrochloride. NCI Thesaurus. Code C47702.

The hydrochloride salt form of remifentanil, a synthetic anilidopiperidine derivative and short-acting opiate agonist with analgesic and anesthetic properties. Remifentanil selectively binds to and activates the mu-opioid receptor, thereby producing analgesia, respiratory depression, miosis, reduced gastrointestinal motility, and euphoria. 